\title{
General Equations for Determining Species Involved in Solution Equilibria and Equilibrium Constants from Solution Molecular Mass Measurements
}

\author{
Matthew G. Davidson, Ronald Snaith, Dietmar Stalke, and Dominic S. Wright* \\ University Chemical Laboratory, Lensfield Road, Cambridge CB2 1EW, U.K.
}

Received August 31, 1992 (Revised Manuscript Received January 26, 1993)

\begin{abstract}
A simple way by which equilibrium species can be identified with reasonable certainty, and equilibrium constants and thermodynamic data can be thereby extracted, from variable-concentration cryoscopic molecular mass measurements in solution is reported. The method relies on the assumption that the individual molecular species involved in such solution equilibria exert independent and additive contributions to the depression in freezing point from that of the pure solvent. Given this assumption, individual equations can be developed for a given dynamic equilibrium, relating the equilibrium constant $\left(K_{\mathrm{B}}\right)$ to the molecular mass $\left(M_{\mathrm{r}}\right)$ and hence to the degree of association $(n)$ of the species involved. The measurements will fit best one such equation and can thereby be used to pinpoint the equilibrium species present. To first illustrate this method, the variation of the degree of association $(n)$ has been examined over a range of concentrations (up to ca. $0.1 \mathrm{~mol} \mathrm{~L}^{-1}$ ) for two lithium-containing complexes in benzene solutions; the inorganic complex, lithium bromide pentamethyldiethylenetriamine, (LiBr.PMDETA $)_{n}$ (1) [PMDETA $=\mathrm{MeN}\left(\mathrm{CH}_{2} \mathrm{CH}_{2} \mathrm{NMe}_{2}\right)_{2}$ ], and the amidolithium reagent, (dicyclohexylamido)lithium hexamethylphosphoramide, $\left[\left(\mathrm{C}_{6} \mathrm{H}_{11}\right)_{2} \mathrm{NLi} \cdot \mathrm{HMPA}\right]_{n}$ (2) [HMPA = $\left(\mathrm{Me}_{2} \mathrm{~N}\right)_{3} \mathrm{P}=0$ ]. While both complexes have known dimeric structures in the solid state, the cryoscopic work presented here, and its fitting to specific equations, implies that both 1 and 2 are involved in essentially dimer $\rightleftharpoons$ monomer equilibria in such solutions. On this basis, the equilibrium constants for both complexes were determined [for $1, K_{\mathrm{s}}=1.41 \times 10^{-2} \mathrm{~mol} \mathrm{~L}^{-1}, \Delta G^{\circ}=9.8 \mathrm{~kJ} \mathrm{~mol}^{-1}$; and for $\left.2, K_{\mathrm{s}}=4.0 \times 10^{-3} \mathrm{~mol} \mathrm{~L}^{-1}, \Delta G^{\circ}=12.8 \mathrm{~kJ} \mathrm{~mol}^{-1}\right]$.
\end{abstract}

\section{Introduction}

Lithium compounds $(\mathrm{LiX})_{n}$ and complexes $(\mathrm{LiX} \cdot x \mathrm{~L})_{n}$ ( $\mathrm{L}=\mathrm{Lewis}$ base), and alkali metal derivatives in general, have aroused considerable interest, especially over the last decade. In major part, this interest has stemmed from their value as synthetic reagents, e.g., especially lithiated organics such as $\mathrm{C}-\mathrm{Li}^{1}$ and $\mathrm{N}-\mathrm{Li}^{2}$ species which are widely used as proton abstractors in numerous organic syntheses. ${ }^{3}$ Most attention has focused on the elucidation of solidstate structures. However, in their role as reagents, the crucial factor is their structure(s) in solution (in hydrocarbons such as benzene and toluene, and in ethereal solvents) where they are actually employed. Several strategies have been used to elucidate solution structures and to provide information about equilibria involved. The solid-state structures of complexes may provide at best an indication of their structures in solution. Variabletemperature and heteronuclear $\left({ }^{1} \mathrm{H},{ }^{13} \mathrm{C},{ }^{6} \mathrm{Li},{ }^{7} \mathrm{Li},{ }^{15} \mathrm{~N}\right) \mathrm{NMR}$ studies ${ }^{4}$ can also give insight into the complicated equilibria which frequently occur. However, to date, the role of cryoscopy and other colligative measurements in these and related studies has largely been qualitative..$^{5}$ That is to say that these measurements have been used merely to give an indication of how the degree of association $(n)$ varies with concentration. By itself, this does not identify which equilibrium species are present (e.g., a fractional value between $n=1$ and $n=2$ could be due to monomer

(1) C-Li compounds: (a) Setzer, W.; Schleyer, P.v. R. Adv. Organomet. Chem. 1985, 24, 353. Na, K, Rb, and $\mathrm{Cs}$ compounds. (b) Schade, C. Schleyer, P. v. R. Adv. Organomet. Chem. 1987, 27, 169.

(2) N-Li and N-Na compounds: (a) Gregory, K.; Schleyer, P. v. R.; Snaith, R. Adv. Inorg. Chem. 1991, 37, 47, (b) Mulvey, R. E. Chem. Soc. Rev. 1991, 20,167. (c) Seebach, D. Angew. Chem. 1988, 100, 1685. Angew. Chem. Int. Ed. Engl. 1988, 27, 1624.

(3) Fieser, M. Reagents for Organic Synthesis; Wiley Interscience: New York, 1990; Vol. 15 and earlier volumes. $\rightleftharpoons$ dimer, or monomer $\rightleftharpoons$ trimer, or solvent-separated ionpair $\rightleftharpoons$ dimer, etc.) and thus cannot afford any quantitative information such as equilibrium constants.

We describe here a cryoscopic approach which identifies with reasonable certainty the solution equilibrium processes occurring and which thereby affords key thermodynamic data [equilibrium constants $\left(K_{\mathrm{s}}\right)$ and Gibb's free energy changes $\left(\Delta G^{\circ}\right)$ ] for such processes. This approach assumes no prior knowledge of the solid-state structure or NMR measurements of the compound. All that is needed is the empirical formula and thereby the formula mass. In essence, it has proved possible to derive simple and specific equations relating the concentration of a species in solution (expressed usually relative to a monomer, i.e., to the empirical formula) to the cryoscopically determined association state $(n)$ in solution. Several equilibrium

(4) See for example, (a) Hall, P. L.; Gilchrist, J. H.; Harrison, A. T. Fuller, D. J.; Collum, D.B.J.Am. Chem. Soc. 1991,113,9575. (b) Kallman N.; Collum, D. B. J. Am. Chem. Soc. 1987, 109, 7466. (c) Galiano-Roth, A. S.; Michaelidis, E. M.; Collum, D. B. J. Am. Chem. Soc. 1988, 110, 2658. (d) DePue, J. S. Collum D. B. J Am. Chem. Sac. 1988, 110, 5518. (e) Günther, H.; Moskau, D.; Bast, P.; Schmalz, D. Angew. Chem. 1987, 99, 1242. Angew. Chem. Int. Ed. Engl. 1987, 26, 1212. (f) Bauer, W.; Clark, T.; Schleyer, P. v. R. J. Am. Chem. Soc. 1987, 109, 970. (g) Hoffmann, D.;Bauer, W.; Schleyer, P.v. R.J.Chem. Soc.Chem. Commun. 1990, 208. (h) Bauer, W.; Winchester, W. R.; Schleyer, P. v. R. Organometallics 1987,6, 2371. (i) Fraenkel, G.; Fraenkel, A. M.; Geckle, M. J.; Schloss, F. J. Am. Chem. Soc. 1979, 101, 4745. (j) Fraenkel, G.; Henrichs, M.; Hewitt, J. M.; Su, B. M.; Geckle, M. J. J. Am. Chem. Soc. 1980, 102, 3345. (k) Fraenkel, G.; Henrichs, M.; Hewitt, J. M.; Su, B. M. J. Am. Chem. Soc. 1984, 106, 255.

(5) See for example, N-Li compounds: (a) Barr, D.; Clegg, W.; Mulvey, R. E.; Reed, D.; Snaith, R. Angew. Chem. 1985, 97, 322. Angew. Chem. Int Ed Engl 1985, 24, 328. (b) Reed, D.; Barr, D.; Mulvey, R. E.; Snaith, R. J. Chem. Soc., Dalton Trans. 1986, 557. (c) Barr, D.; Mulvey, R. E.; Snaith, R.; Wade, K.; Reed, D. Magn.Reson.Chem. 1986, 24,713. Selected C-Li compounds: (d) Lewis, H. L.; Brown, T. L. J. Am. Chem. Soc. 1970, 92, 1859. (e) Brown, T. L.; Rogers, M. T. J. Am. Chem. Soc. 1957, 79, 1859. Selected C-Mg compounds, (f) Ashby, E. C.; Becker, W. E. J. Am. Chem. Soc. 1963, 85, 118. (g) Ashby, E. C.; Smith, M. B. J. Am. Chem. Soc. $1964,86,4363$. 


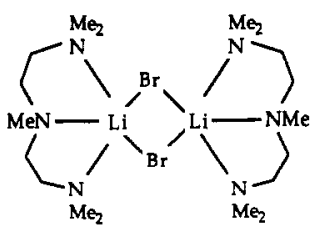

(1)

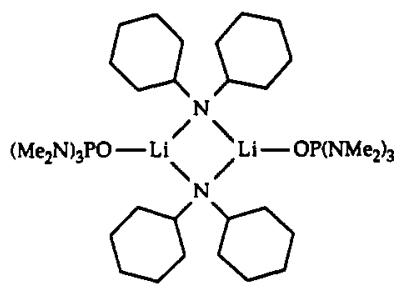

(2)
Figure 1. Dimeric solid-state structures of 1 and 2.

situations are considered, e.g., dimer $\rightleftharpoons$ monomer, trimer $\rightleftharpoons$ dimer + monomer, contaction-pair $\rightleftharpoons$ solvent-separated ion-pair etc., and the corresponding equations given. Other equations for other equilibria can readily be worked out using principles described here (see Theory). Each equation can be plotted as a curve, the upper limit of which defines the association state $(n)$ of the species dissociating, e.g., $n$ approaches 3 for a trimer $(\mathrm{AB})_{3}$ as the concentration approaches $\infty$. The lower limit of the curve (as the concentration approaches zero) and the precise shape of the curve between the upper and lower limits depend on the actual dissociative equilibrium which occurs, e.g., from a trimer $(\mathrm{AB})_{3}$, one can envisage formation of dimers ( $n$ tending toward 2 at low concentration), of monomers + dimers $(n \rightarrow 1.5)$, of monomers alone $(n \rightarrow 1)$, or of separated ion-pairs $(n \rightarrow 0.5)$. Cryoscopic data obtained from a solution in which an equilibrium occurs will best fit an equation specific to the equilibrium occurring, and thus the nature of the equilibrium occurring can be established with reasonable certainty. Additionally, once such a nature has been established, further analysis of the cryoscopic results yields thermodynamic data $\left(K_{\mathrm{s}}\right.$ and $\Delta G^{\circ}$ values). Although in the past colligative measurements (notably, ebullioscopy and vapor phase osmometry) have been used quantitatively to determine certain equilibrium constants occurring for solution equilibria, such instances have invariably relied on tailoring the methods to individual systems and have involved detailed assumptions. ${ }^{6}$ The latter prevent their application to any unrelated systems. In this work we present a simple method, requiring no restrictive assumptions, which for the first time is applied to cryoscopy-a method of colligative measurement accessible to, and extensively employed by, chemists.

To exemplify this treatment, the inorganic complex lithium bromide pentamethyldiethylenetriamine, (Li$\mathrm{Br} \cdot \mathrm{PMDETA})_{n}(1)\left[\mathrm{PMDETA}=\mathrm{MeN}\left(\mathrm{CH}_{2} \mathrm{CH}_{2} \mathrm{NMe}_{2}\right)_{2}\right]^{7}$ and the organic reagent (dicyclohexylamido)lithium hexamethylphosphoramide, $\left[\left(\mathrm{C}_{6} \mathrm{H}_{11}\right)_{2} \mathrm{NLi} \cdot \mathrm{HMPA}\right]_{n}$ (2) $\left[\mathrm{HMPA}=\left(\mathrm{Me}_{2} \mathrm{~N}\right)_{3} \mathrm{P}=0\right]^{5 \mathrm{~b}, 8}$ have been examined. Both 1 and 2 are in fact known to be dimers [ $n=2]$ in the solid state, having $(\mathrm{LiBr})_{2}$ and $(\mathrm{LiN})_{2}$ rings $^{8}$ respectively (Figure 1).

Cryoscopic measurements have been employed, over a range of concentrations (up to $0.1 \mathrm{~mol} \mathrm{~L}^{-1}$ ) in benzene, firstly to establish the probable general nature of any

(6) (a) Walker, F. W.; Ashby, E. C. J. Am. Chem. Soc. 1969, 91, 3845. (b) Schrier, E. E. J. Chem. Ed. 1968, 45, 176. (c) Davies, M.; Thomas, D. K. Phys. Chem. 1956, 60, 763. (d) Tso'o, P. O. P.; Melvin, I. S.; Olson A. C. J. Am. Chem. Soc. $1963,85,1289$.

(7) Hall, S. R.; Raston, C. L.; Skelton, B. W.; White, A. H. Inorg. Chem. 1983, 22, 4070 .

(8) Although, as stressed in the introduction, solid-state structures are incidental to results obtained by this method, during the course of the study the structure of 2 was determined by X-ray diffraction and shown to be dimeric in the solid state. A detailed discussion of the structure is not given here. equilibria occurring for 1 and 2 in solution, and then to derive equilibrium constants and corresponding Gibb's free energy changes ( $K_{\mathrm{s}}$ and $\Delta G^{\circ}$, respectively). Complex 1 has not previously been examined in solution, though for 2 limited cryoscopic data have been reported and, linked to ${ }^{7} \mathrm{Li} \mathrm{NMR}$ spectroscopic results, used to suggest that a dimer $\rightleftharpoons$ monomer equilibrium operates. ${ }^{5 b}$ Our cryoscopic results indicate that a simple dimer $\rightleftharpoons$ monomer equilibrium occurs over all concentrations examined for 1 in solution, with $K_{\mathrm{g}}=1.41 \times 10^{-2} \mathrm{~mol} \mathrm{~L}^{-1}$ and $\Delta G^{\circ}=9.8 \mathrm{~kJ}$ $\mathrm{mol}^{-1}$. However, for 2, while a dimer $\rightleftharpoons$ monomer equilibrium is apparent at relatively higher concentrations (with $K_{\mathrm{g}}=4.0 \times 10^{-3} \mathrm{~mol} \mathrm{~L}^{-1}$ and $\Delta G^{\circ}=12.8 \mathrm{~kJ} \mathrm{~mol}^{-1}$ ), a more complicated fragmentational equilibrium is revealed from measurements obtained at lower concentrations $\left(<0.02 \mathrm{~mol} \mathrm{~L}^{-1}\right)$.

\section{Theory}

It is known that the depression in freezing point $(\Delta T)$ is related to the mass of solute $\left(w_{\mathrm{s}}\right)$ of molecular mass $\left(M_{\mathrm{r}}\right)$ dissolved in a solvent of mass $\left(w_{\mathrm{b}}\right)$ by the equation

$$
\Delta T=\frac{1000 K_{\mathrm{b}} w_{\mathrm{s}}}{w_{\mathrm{b}} M_{\mathrm{r}}}
$$

where $K_{\mathrm{b}}$ is the cryoscopic constant. ${ }^{9}$

If it is assumed that in an equilibrium process each of the species involved exerts an independent (and additive) contribution to the freezing point depression compared to the freezing point of the pure solvent, then

$$
\frac{w_{\mathrm{s}}}{M_{\mathrm{r}}}=\sum_{i} \frac{w_{i}}{m_{i}}
$$

Consider the case of an equilibrium involving one mole of species 1 dissociating into equimolar amounts of each of species 2 and 3 :

$$
1 \rightleftharpoons 2+3
$$

Then the total mass of solute $\left(w_{\mathrm{g}}\right)$ is given by

$$
w_{s}=w_{1}+w_{2}+w_{3}
$$

and, from eq 2 ,

$$
\frac{w_{\mathrm{s}}}{M_{\mathrm{r}}}=\frac{w_{1}}{m_{1}}+\frac{w_{2}}{m_{2}}+\frac{w_{3}}{m_{3}}
$$

However, the concentration of species $2\left(C_{2}\right)$ equals the concentration of species $3\left(C_{3}\right)$, and thus from eq 5 ,

$$
\frac{w_{\mathrm{s}}}{M_{\mathrm{r}}}=\frac{w_{1}}{m_{1}}+\frac{2 w_{2}}{m_{2}}
$$

A coefficient $\alpha$, corresponding to the molecular mass fraction of species 1 which dissociates to species 2 , can then be defined, e.g., for a pentamer 1 of molecular mass 100 giving a trimer 2 (mass 60) plus a dimer 3 (mass 40), $\alpha=0.60$. Hence,

$$
\alpha m_{1}=m_{2}
$$

when eq 6 becomes

(9) (a) Atkins, P. W. Physical Chemistry, 2nd ed.; Oxford University Press: Oxford, 1983. (b) Moore, M. J. Physical Chemistry, 5th ed.; Longman: London, 1976. (c) Adam, N. K. Physical Chemistry; Oxford University Press: Oxford, 1956. (d) Gordon, A. J.; Ford, A. R. The Chemists Companion; A Handbook of Practical Data, Techniques and References; Wiley: New York, 1972. 


$$
\frac{w_{\mathrm{s}}}{M_{\mathrm{I}}}=\frac{w_{1}}{m_{1}}+\frac{2}{\alpha}\left(\frac{w_{2}}{m_{1}}\right)
$$

However, if from eq $7 \alpha m_{1}=m_{2}$, then $(1-\alpha) m_{1}=m_{3}$ and so

$$
\frac{\alpha}{1-\alpha}=\frac{m_{2}}{m_{3}}
$$

From eq 4 and the fact that $C_{2}=C_{3}$,

$$
w_{\mathrm{s}}=w_{1}+w_{2}+\frac{m_{3}}{m_{2}} w_{2}
$$

Then, in combination with eq 9 , eq 10 becomes

$$
w_{\mathrm{s}}=w_{1}+w_{2}+\frac{1-\alpha}{\alpha} w_{2}
$$

so that

$$
w_{\mathrm{s}}=w_{1}+\frac{w_{2}}{\alpha}
$$

Eq 8 transposes to

$$
w_{\mathrm{s}}=\frac{M_{\mathrm{r}}}{m_{1}}\left(w_{1}+\frac{2 w_{2}}{\alpha}\right)
$$

and then, substituting for $w_{1}$ using eq 12 ,

$$
\begin{gathered}
w_{\mathrm{s}}=\frac{M_{\mathrm{r}}}{m_{1}}\left(w_{\mathrm{s}}-\frac{w_{2}}{\alpha}+\frac{2 w_{2}}{\alpha}\right) \\
w_{\mathrm{s}}=\frac{M_{\mathrm{r}}}{m_{1}}\left(w_{\mathrm{s}}+\frac{w_{2}}{\alpha}\right) \\
w_{2}=\alpha w_{\mathrm{s}}\left(\frac{m_{1}}{M_{\mathrm{r}}}-1\right)
\end{gathered}
$$

Eq 12 transposes to

$$
w_{1}=w_{\mathrm{g}}-\frac{w_{2}}{\alpha}
$$

Substituting for $w_{2}$ from eq 14 gives

$$
\begin{gathered}
w_{1}=w_{\mathrm{s}}-\left(\frac{1}{\alpha}\right) \cdot \alpha \cdot w_{\mathrm{s}}\left(\frac{m_{1}}{M_{\mathrm{r}}}-1\right) \\
w_{1}=w_{\mathrm{s}}\left(2-\frac{m_{1}}{M_{\mathrm{r}}}\right)
\end{gathered}
$$

Expressions for the concentrations can then be deduced:

$$
C_{1}=\frac{w_{1}}{m_{1} V}
$$

where $V$ is the volume of solution. Substituting for $w_{1}$ from eq 15 gives

$$
C_{1}=\frac{w_{\mathrm{s}}}{m_{1} V}\left(2-\frac{m_{1}}{M_{\mathrm{r}}}\right)
$$

Similarly

$$
C_{2}=\frac{w_{2}}{m_{2} V}
$$

Substituting for $w_{2}$ from eq 14 gives

$$
C_{2}=\frac{\alpha w_{\mathrm{s}}}{m_{2} V}\left(\frac{m_{1}}{M_{\mathrm{r}}}-1\right)
$$

The total concentration of solute, $C_{8}$, expressed in terms of the species (1) dissociating, is given by

$$
C_{\mathrm{s}}=\frac{w_{\mathrm{s}}}{m_{1} V}
$$

Also, from eq 7 ,

$$
\frac{\alpha}{m_{2}}=\frac{1}{m_{1}}
$$

Hence eq 16 becomes

$$
C_{1}=C_{\mathrm{s}}\left(2-\frac{m_{1}}{M_{\mathrm{r}}}\right)
$$

and eq 17 becomes

$$
C_{2}=C_{\mathrm{s}}\left(\frac{m_{1}}{M_{\mathrm{s}}}-1\right)
$$

Eqs 18 and 19 can then be substituted into the usual equilibrium constant expression for equilibrium eq 3 , noting that $C_{2}=C_{3}$ :

$$
K_{\mathrm{s}}=\frac{C_{\mathrm{s}}\left(\frac{m_{1}}{M_{\mathrm{r}}}-1\right)^{2}}{\left[1-\left(\frac{m_{1}}{M_{\mathrm{r}}}-1\right)\right]}
$$

Since $n=M_{\mathrm{r}} / m_{1}$ and $m_{1} / M_{\mathrm{r}}=1 / n$, then

$$
K_{\mathrm{s}}=\frac{C_{\mathrm{s}}\left(\frac{1}{n}-1\right)^{2}}{\left[1-\left(\frac{1}{n}-1\right)\right]}
$$

so

$$
K_{\mathrm{s}}=\frac{C_{\mathrm{s}}\left(\frac{1-n}{n}\right)^{2}}{\left(\frac{2 n-1}{n}\right)}
$$

and

$$
K_{\mathrm{s}}=\frac{C_{\mathrm{s}}(1-n)^{2}}{n(2 n-1)}
$$

The equation $\Delta G^{\circ}=-R T$ n $K_{\mathrm{s}}$ can then be used to give the Gibb's free energy $\left(\Delta G^{\circ}\right)$ where $n=$ degree of association defined in terms of the species dissociating and $C_{\mathrm{B}}=$ concentration of the species dissociating.

The general eq 20 can be applied to any equilibrium reaction in which one mole of the species dissociating [species 1 in eq 3] affords equimolar amounts of two "products" (species 2 and 3, which might or might not be the same). However, the principles outlined above allow construction of general equations for other situations, e.g., an equilibrium in which equimolar amounts of products are not afforded, such as tetramer $\rightleftharpoons$ dimer +2 (monomers); e.g., an equilibrium affording three (or more) products, such as hexamer $\rightleftharpoons$ trimer + dimer + monomer. For such other situations, the key point is that the concentrations of the products are not independent and thus there remain effectively just two unknowns: the concentration of the species dissociating and the concentration of one product. An equation for such a situation 
Table I. Derived Equations for Particular Equilibrium Types

\begin{tabular}{cc}
\hline type & equilibrium equation \\
\hline $\mathrm{A}$ (general dissociation) \\
$\mathrm{AB} \rightleftharpoons \mathrm{A}+\mathrm{B}$ & $K_{\mathrm{s}}=\frac{C_{\mathrm{s}}(1-n)^{2}}{n(2 n-1)}$ \\
$\mathrm{B}($ oligomer dissociation) \\
$\mathrm{A}_{m} \rightleftharpoons m \mathrm{~A}$ & $K_{\mathrm{s}}=\frac{C_{\mathrm{s}}^{m-1}(m-n)^{m}}{n^{m-1}(m-1)^{m-1}(\mathrm{n}-1)}$ \\
$\mathrm{C}$ (oligomer dissociation) \\
$\mathrm{A}_{m} \rightleftharpoons 2 \mathrm{~A}_{m / 2}$ & $K_{\mathrm{s}}=\frac{4 C_{\mathrm{s}}(m-n)^{2}}{n m(2 n-m)}$
\end{tabular}

can then be constructed, relating $K_{\mathrm{s}}$ to the two quantities known from the experiment $\left(C_{8}\right.$, the concentration of the dissolved material, expressed in some suitable way, and $n$, the averaged aggregation state of the dissolved material).

\section{Applications of the General Equation (see Table I)}

The general eq 20 is applicable as it stands to equilibria of type: $\mathrm{AB} \rightleftharpoons \mathrm{A}+\mathrm{B}$, e.g., $\mathrm{CrownM}^{+} \cdot \mathrm{X}^{-} \rightleftharpoons \mathrm{CrownM}^{+}+$ $\mathrm{X}^{-}$.

For other equilibria of general type $1 \rightleftharpoons 2+3$ (eq 3 ), it is then usual and convenient to express $C_{8}$ and $n$ in terms of a monomer, i.e., of the empirical formula. For example, for a dimer $\rightleftharpoons$ monomer equilibrium, $\mathrm{A}_{2} \rightleftharpoons 2 \mathrm{~A}$. $C_{\mathrm{s}}$ [in eq 20, the concentration of the species dissociating] becomes $2 C_{8}$ (where $C_{\mathrm{s}}$ is now the concentration of the monomer, A) and $n$ becomes $n / 2$ (now the degree of association defined in terms of the monomer).

$$
K_{\mathrm{s}}=\frac{C_{\mathrm{s}}(1-n)^{2}}{n(2 n-1)}
$$

becomes

$$
\begin{gathered}
K_{\mathrm{s}}=\frac{2 C_{\mathrm{s}}\left(1-\frac{n}{2}\right)^{2}}{\frac{n}{2}(n-1)} \\
K_{\mathrm{s}}=\frac{4 C_{\mathrm{s}} \cdot \frac{(2-n)^{2}}{4}}{n(n-1)} \\
K_{\mathrm{s}}=\frac{C_{\mathrm{s}}(2-n)^{2}}{n(n-1)}
\end{gathered}
$$

Eq 21 is a specific case ( $m=2$ ) of general equations for oligomer dissociation into monomers, i.e., $\mathbf{A}_{m} \rightleftharpoons m \mathrm{~A}$ or into monomers, or dimers, or tetramers, etc., i.e., $\mathbf{A}_{m} \rightleftharpoons$ $2 \mathrm{~A}_{m / 2}$ (see Table I).

The general eq 20 can also deal with equilibria of an oligomer into two other distinct oligomers:

$$
\text { e.g., } \underset{\text { pentamer }}{\mathrm{A}_{6}} \rightleftharpoons \underset{\text { trimer }}{\mathrm{A}_{3}}+\underset{\text { dimer }}{\mathrm{A}_{2}}
$$

In terms of a monomer (A), $C_{8}$ becomes $5 C_{8}$ and $n$ becomes $n / 5$. Substituting these transposed quantities into eq 20 gives

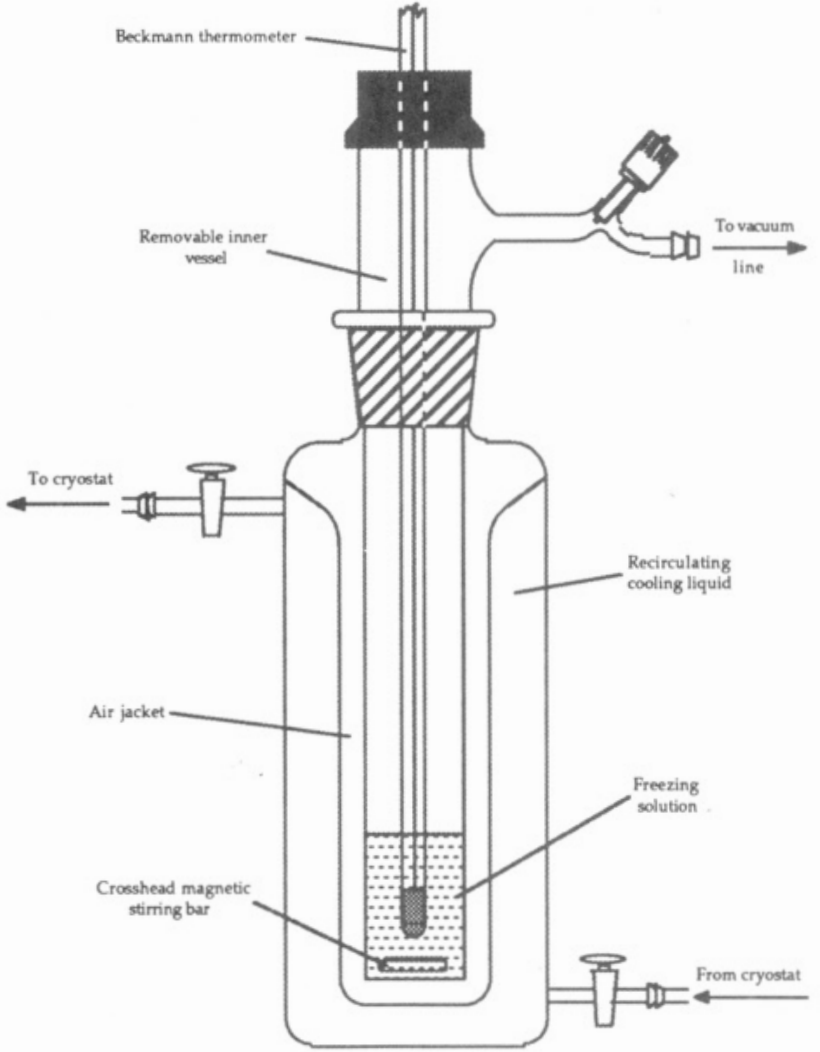

Figure 2. Diagram of cryoscopy apparatus.

$$
K_{\mathrm{s}}=\frac{25 C_{\mathrm{s}}\left(1-\frac{n}{5}\right)^{2}}{n\left(\frac{2 n}{5}-1\right)}
$$

It can be seen from eq 22 that at high concentration $\left(C_{s}\right.$ $\rightarrow \infty$, the upper limit of a plot of this equation) when $n$ $\rightarrow 5$, i.e., the undissociated pentamer is the major solution species, then $K_{\mathrm{s}} \rightarrow 0$. Similarly, at low concentration, $n$ $\rightarrow 2.5$ (each mole of pentamer having largely dissociated into one mole each of trimer + dimer), and so $K_{8} \rightarrow \infty$.

\section{Experimental Section}

General Procedures. Standard inert atmosphere techniques ${ }^{10}$ were used for the preparations and subsequent investigations of the compounds described.

Syntheses. Crystalline samples of $(\mathrm{LiBr} \cdot \mathrm{PMDETA})_{n}(1)$ were prepared by the reaction of ammonium bromide, suspended in a solution of toluene and PMDETA, with $n$-butyllithium (1:1:1 equiv) ${ }^{11}$ and were stored under nitrogen in a glove box prior to use. Crystals of $\left[\left(\mathrm{C}_{6} \mathrm{H}_{11}\right)_{2} \mathrm{NLi} \cdot \mathrm{HMPA}\right]_{n}(2)$ were prepared, in the manner described previously, ${ }^{5 \mathrm{~b}}$ freshly before each cryoscopic run in order to minimize oxidation and hydrolysis by air and moisture during storage.

Cryoscopic Determinations. The specially designed cryoscopic apparatus (shown in Figure 2) is comprised of a flatbottomed inner glass sample tube surrounded by an outer cooling jacket filled with circulating ethanol. An air jacket separates the inner tube from the outer cooling jacket and prevents supercooling of the solution.

The temperature of the cooling jacket is controlled by a cryostat at just $\left(\right.$ ca. $1^{\circ} \mathrm{C}$ ) below the freezing point of benzene. A side arm fitted with a tap allowed a dry, oxygen-free nitrogen atmosphere to be maintained during measurements. The depression in

(10) Schriver,D.F.; Drezdzon, M.A.The Manipulation of Air-Sensitive Compounds, 2nd ed.; Wiley: New York, 1986.

(11) Barr, D.; Snaith, R.; Wright, D. S.; Mulvey, R. E.; Wade, K. J. Am. Chem. Soc. 1987, 109, 7891 . 
freezing point $(\Delta T)$ of each solution, in which known quantities of the complexes were present, were recorded using a Beckmann thermometer (reading to $0.001^{\circ} \mathrm{C}$ with the aid of an eyepiece). This fits into the inner jacket with a tight seal so that the thermometer bulb is positioned midway into the measured solution. The solutions were stirred with a magnetic vortex stirrer in order to prevent supercooling and to ensure that an average temperature was recorded for every reading. Benzene specifically purified for molecular mass determinations ${ }^{12}$ was used in these determinations and was further dried using molecular sieves. The benzene was regularly standardized by determining the cryoscopic constant $\left(K_{\mathrm{b}}\right)$ from $\Delta T$ values of known concentrations of solutions of freshly sublimed biphenyl $\left[\left(\mathrm{C}_{6} \mathrm{H}_{5}\right)_{2}, M_{\mathrm{r}}=154.21\right]$ throughout these investigations. Initially, the freezing point of pure benzene was determined before a known mass of the complex was introduced into the apparatus. The sample was weighed out in an air-tight vial in a glove box and introduced under a stream of nitrogen into the apparatus. The exact mass added was determined by difference. The freezing point of the resulting solution was then determined and hence the freezing point depression $(\Delta T)$. From the cryoscopic equation [eq 1, expressed in terms of $M_{\mathrm{r}}$ ], the molecular mass was then calculated.

\section{Results and Discussion}

The cryoscopic equations given here (Theory and Table I) represent some of the most commonly occurring situations found for main group inorganic and organometallic compounds; in principle, similar equations can be derived for any equilibrium situation. Equilibria of type A represent for example situations in which ionpairing and ion-separation occurs; such frequently arise e.g. with crown ether inorganic salt complexes in solution. ${ }^{13}$ Equilibria of types B and C define typical oligomeric dissociation processes commonly observed in organometallic systems (such as lithium, aluminum, and magnesium organometallics) which are widely employed as synthetic reagents in solution. ${ }^{1-3}$ All these equilibrium equations can be plotted as curves whose lower limit of $n$ (as $C_{\mathrm{s}} \rightarrow$ 0 ) is given by the denominator and whose upper limit of $n\left(\right.$ as $\left.C_{8} \rightarrow \infty\right)$ is given by the numerator of each expression. Of importance here is that the curvature and exact form, as well as the lower and upper limits, of the plot of each equation type give a simple diagnostic way by which the equilibrium occurring can be deduced in an individual case. This point is stressed in Figure 3 which shows the relationship between degree of association $(n)$ and formal concentration $\left(C_{8}\right)$ for different oligomers dissociating into monomers [Table $\mathrm{I}$, type $\mathrm{B}, m=2-6$; the equilibrium constants for all four examples are set (arbitrarily) at $1 \times 10^{-3}$.

An important point, relating degree of association $(n)$ and the reactivity of a reagent in solution, can be illustrated by the simple example of a dimer $\rightleftharpoons$ monomer equilibrium. The predicted variation in degree of association $(n)$ with concentration and with dissociative equilibrium constants $K_{8}$ (according to eq 21) is shown in Figure 4. In Figure 4, the horizontal line at $n=1.33$ corresponds to $50 \%$ dissociation of dimers into monomers. Consequently, complexes with equilibrium constants $\left(K_{\mathrm{s}}\right)$ above $10^{-1} \mathrm{~mol}$ $\mathrm{L}^{-1}$ exist mainly as more reactive monomers over a wide range of concentrations, whereas those with $K_{\mathrm{B}}$ below $10^{-1}$

(12) Cryoscopic grade benzene was obtained from Fisons Scientific Equipment, Bishop Meadow Road, Loughborough, Leicestershire, LE11 ORG, U.K.

(13) (a) Alfheim, T.; Dale, J.; Groth, P.; Kraufwurst, K. D. J. Chem. Soc. Chem. Commun. 1984, 1502. (b) Mathieu, F.; Metz, B.; Moros, D. Weiss, R. J. Am. Chem. Soc. 1978, 100, 4412 . (c) Miller, J. M.; Clark, J. H. J. Chem. Soc. Chem. Commun. 1982, 1318

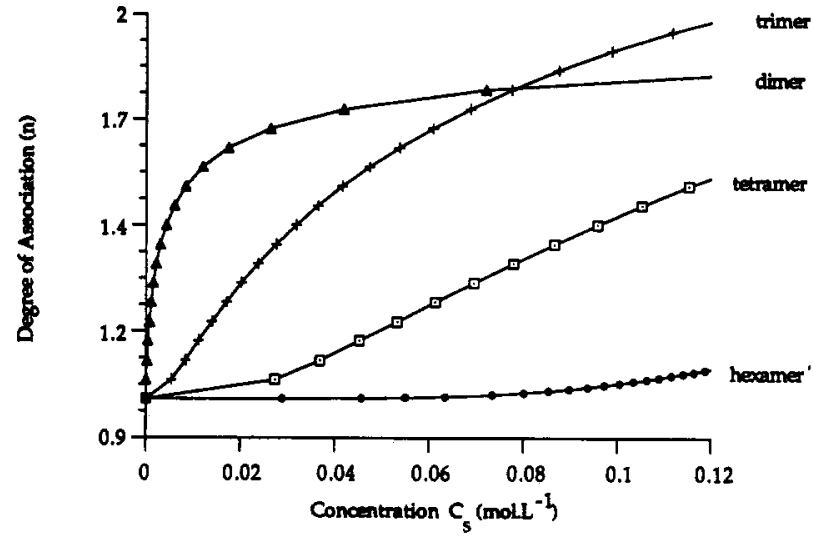

Figure 3. Theoretical variation of degree of association $(n)$ with concentration $\left(C_{\mathrm{s}}\right)$ for various oligomer $\rightleftharpoons$ monomer equilibrium processes with the same equilibrium constant $\left(K_{\mathrm{s}}=1 \times 10^{-3}\right)$.

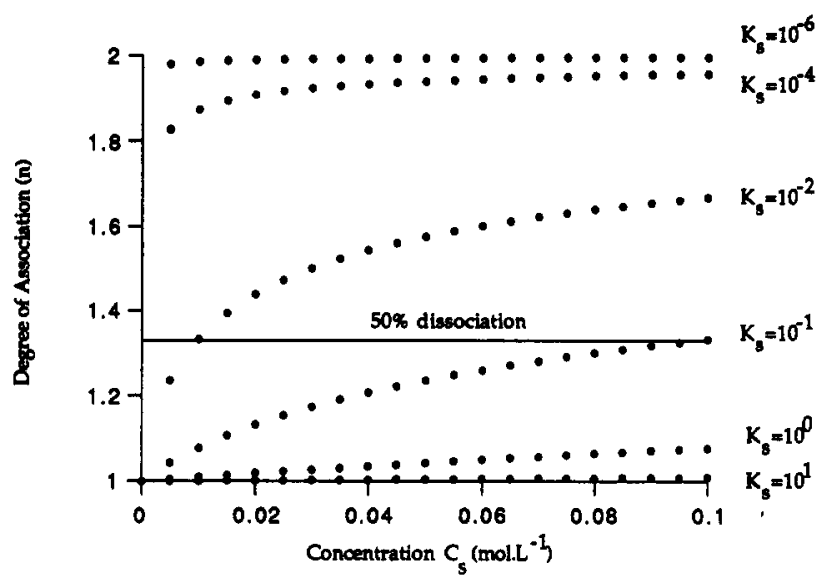

Figure 4. Variation of degree of association $(n)$ with concentration $\left(C_{\mathrm{s}}\right)$ and equilibrium constant $\left(K_{\mathrm{s}}\right)$ for a dimer $\rightleftharpoons$ monomer equilibrium.

Table II. Cryoscopic Molecular Mass Measurements on (LiBr.PMDETA) (1)

\begin{tabular}{|c|c|c|c|}
\hline $\begin{array}{c}\text { concentration } \\
\left(C_{8}\right)\left(\mathrm{mol} \mathrm{L}^{-1}\right)\end{array}$ & $\begin{array}{c}\text { depression } \\
\text { in freezing } \\
\text { point }(\Delta T)\left({ }^{\circ} \mathrm{C}\right)\end{array}$ & $\begin{array}{l}\text { molecular } \\
\text { mass }\left(M_{\mathrm{r}}\right)\end{array}$ & $\begin{array}{c}\text { degree of } \\
\text { association }(n)\end{array}$ \\
\hline 0.009 & $0.040 \pm 0.002$ & $360 \pm 18$ & $1.38 \pm 0.07$ \\
\hline 0.012 & $0.050 \pm 0.002$ & $360 \pm 15$ & $1.38 \pm 0.06$ \\
\hline 0.012 & $0.056 \pm 0.002$ & $330 \pm 13$ & $1.27 \pm 0.05$ \\
\hline 0.020 & $0.087 \pm 0.002$ & $351 \pm 8$ & $1.35 \pm 0.03$ \\
\hline 0.026 & $0.107 \pm 0.002$ & $375 \pm 7$ & $1.44 \pm 0.03$ \\
\hline 0.034 & $0.126 \pm 0.002$ & $393 \pm 6$ & $1.51 \pm 0.02$ \\
\hline 0.050 & $0.188 \pm 0.002$ & $391 \pm 5$ & $1.50=0.02$ \\
\hline 0.051 & $0.201 \pm 0.002$ & $395 \pm 5$ & $1.52 \pm 0.02$ \\
\hline 0.077 & $0.294 \pm 0.002$ & $402 \pm 3$ & $1.55 \pm 0.01$ \\
\hline 0.080 & $0.279 \pm 0.002$ & $427 \pm 3$ & $1.64 \pm 0.01$ \\
\hline 0.107 & $0.381 \pm 0.002$ & $432 \pm 3$ & $1.66 \pm 0.01$ \\
\hline
\end{tabular}

mol L $\mathrm{L}^{-1}$ are predominantly dimeric except at very low concentrations. Thus, the magnitude of the equilibrium constant may provide some measure of the potency of a particular reagent (e.g., as a proton abstractor) and the variation of this with concentration. The general equations (Table I) provide thereby a means by which likely activity can be predicted for various equilibria.

Cryoscopic investigations were performed on the inorganic complex (LiBr.PMDETA $)_{n}$ (1) and the amidolithium complex $\left[\left(\mathrm{C}_{6} \mathrm{H}_{11}\right)_{2} \mathrm{NLi} \cdot \mathrm{HMPA}\right]_{n}(2)$ up to concentrations of ca. $0.1 \mathrm{~mol} \mathrm{~L}^{-1}$ in benzene solutions. The results are shown in Tables II and III, respectively. Figures 5 and 6 show the experimental data for compounds 1 and 2 , respectively, in the form of graphs of degree of association 


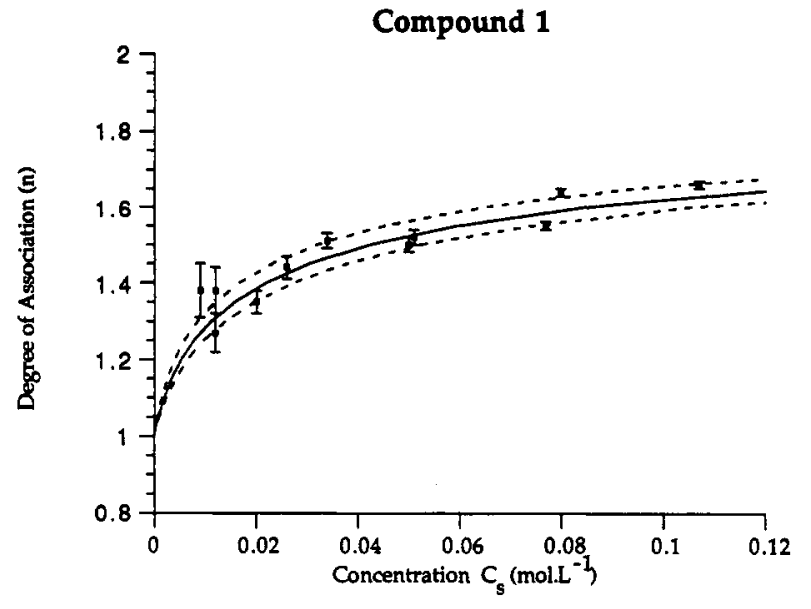

Figure 5. Variation of degree of association $(n)$ with concentration $\left(C_{\mathrm{s}}\right)$ for compound 1 .

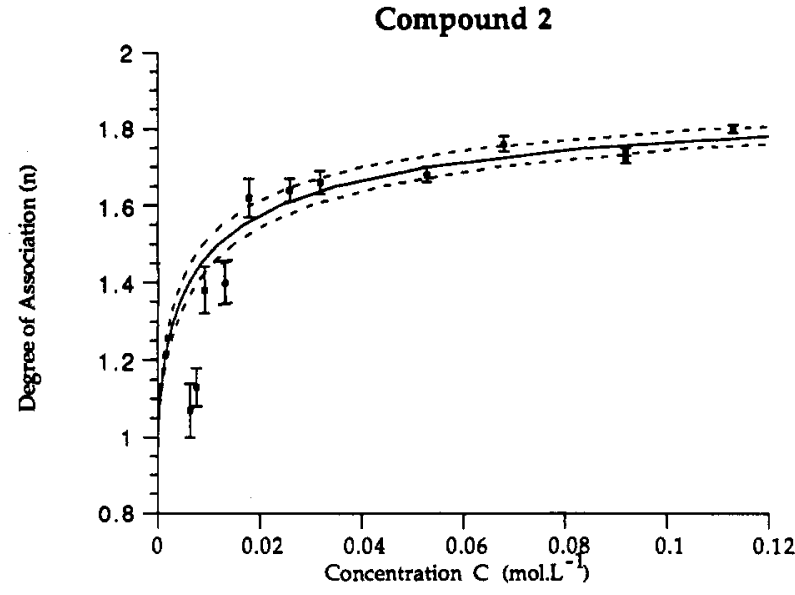

Figure 6. Variation of degree of association $(n)$ with concentration $\left(C_{\mathrm{B}}\right)$ for compound 2.

Table III. Cryoscopic Molecular Mass Measurements on $\left[\left(\mathrm{C}_{6} \mathrm{H}_{11}\right)_{2} \mathrm{NLi} \cdot \mathbf{H M P A}\right]_{n}(2)$

\begin{tabular}{|c|c|c|c|}
\hline $\begin{array}{c}\text { concentration } \\
\left(C_{\mathrm{s}}\right)\left(\mathrm{mol} \mathrm{L}^{-1}\right)\end{array}$ & $\begin{array}{c}\text { depression } \\
\text { in freezing } \\
\text { point }(\Delta T)\left({ }^{\circ} \mathrm{C}\right)\end{array}$ & $\begin{array}{l}\text { molecular } \\
\text { mass }\left(M_{\mathrm{r}}\right)\end{array}$ & $\begin{array}{c}\text { degree of } \\
\text { association }(n)\end{array}$ \\
\hline $\begin{array}{l}6.44 \times 10^{-3} \\
7.76 \times 10^{-3} \\
9.24 \times 10^{-3} \\
1.21 \times 10^{-2} \\
0.018 \\
0.026 \\
0.032 \\
0.053 \\
0.068 \\
0.092 \\
0.092 \\
0.113\end{array}$ & $\begin{array}{l}0.036=0.002 \\
0.041 \pm 0.002 \\
0.054=0.002 \\
0.051 \pm 0.002 \\
0.064 \pm 0.002 \\
0.095 \pm 0.002 \\
0.114 \pm 0.002 \\
0.187 \geq 0.002 \\
0.228 \pm 0.002 \\
0.310=0.002 \\
0.319 \pm 0.002 \\
0.372 \pm 0.002\end{array}$ & $\begin{array}{l}390 \pm 30 \\
412 \pm 21 \\
507 \pm 19 \\
513 \pm 20 \\
593 \geq 19 \\
601 \pm 12 \\
606 \pm 11 \\
616 \pm 7 \\
643 \geq 7 \\
638 \pm 4 \\
630 \pm 4 \\
659 \pm 4\end{array}$ & $\begin{array}{l}1.07 \pm 0.07 \\
1.13 \pm 0.05 \\
1.38 \pm 0.06 \\
1.40 \pm 0.05 \\
1.62 \pm 0.05 \\
1.64 \pm 0.03 \\
1.66 \pm 0.03 \\
1.68 \pm 0.02 \\
1.76 \pm 0.02 \\
1.74 \pm 0.01 \\
1.72 \pm 0.01 \\
1.80 \pm 0.01\end{array}$ \\
\hline
\end{tabular}

Table IV. Calculated Equilibrium Constants $\left(K_{8}\right)$ and Gibb's Free Energies of Dissociation $\left(\Delta G^{\circ}\right)$ for 1 and 2

\begin{tabular}{ccc}
\hline complex & $\begin{array}{c}\text { equilibrium } \\
\text { constant }\left(K_{\mathrm{s}}\right)\left(\mathrm{mol} \mathrm{L}^{-1}\right)\end{array}$ & $\begin{array}{c}\text { dissociative } \\
\Delta G^{\circ}\left(\mathrm{kJ} \mathrm{mol}^{-1}\right)\end{array}$ \\
\hline 1 & $1.41 \pm 0.34 \times 10^{-2}$ & $9.8 \pm 0.7$ \\
2 & $4.0 \pm 1.0 \times 10^{-3}$ & $12.8 \pm 0.6$
\end{tabular}

$(n)$ against the formal concentration of monomer $\left(C_{\mathrm{s}}\right)$ in which best fit theoretical curves (solid lines) have been overlaid for each set of data points. ${ }^{14}$ As indicated in Table IV, the apparent (average) equilibrium constants $\left(K_{\mathrm{s}}\right)$ can be determined from such data, as can the $\Delta G^{\circ}$ values.

The experimental data fit best only one theoretical curve
Scheme I Dissociation in Solution of Dimer 1

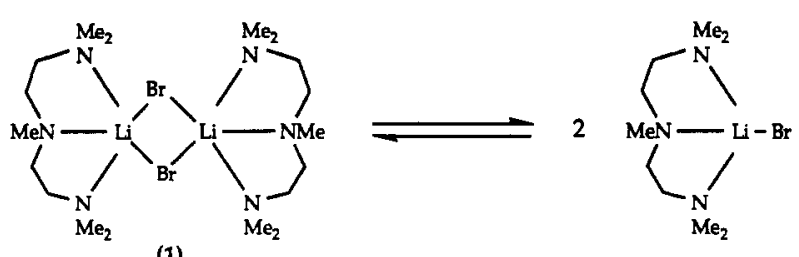

(1)

Scheme II. Dissociation in Solution of Dimer 2

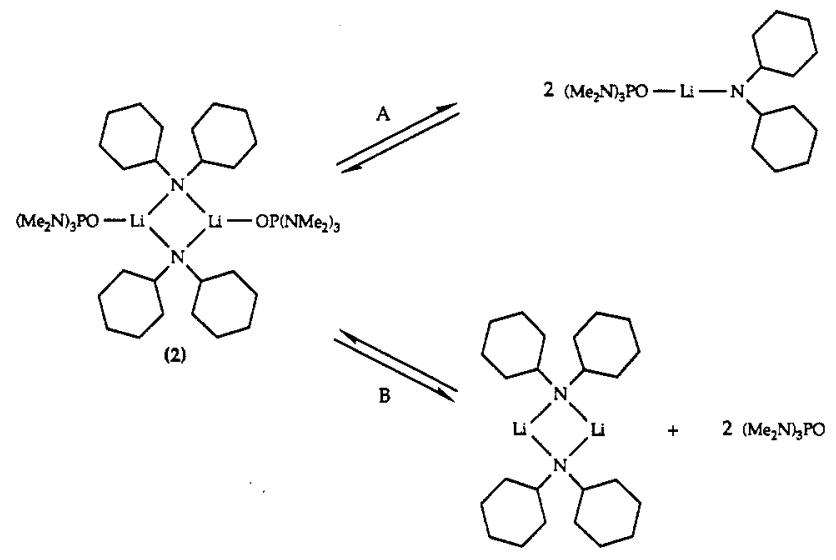

(that of a dimer $\rightleftharpoons$ monomer equilibrium), implying that both complexes engage in essentially dimer $\rightleftharpoons$ monomer equilibria in solution (Schemes I and II for dimers 1 and 2, respectively). For complex 1, the equilibrium constant $\left(K_{\mathrm{s}}\right)$ is $1.41 \pm 0.34 \times 10^{-2} \mathrm{~mol} \mathrm{~L}^{-1}$, corresponding to a value for $\Delta G^{\circ}$ of $9.8 \pm 0.7 \mathrm{~kJ} \mathrm{~mol}^{-1}$. This value compares with $K_{\mathrm{8}}=4.0 \pm 1.0 \times 10^{-3} \mathrm{~mol} \mathrm{~L}^{-1}$ for the amidolithium complex 2 , in which the dissociative $\Delta G^{\circ}$ is $12.8 \pm 0.6 \mathrm{~kJ} \mathrm{~mol}^{-1}$. The differences in the equilibrium constants and free energies of dissociation between 1 and 2 presumably reflect the weakness of $\mathrm{Li}-\mathrm{Br}$ compared to $\mathrm{Li}-\mathrm{N}$ bonds as well as the differing coordination numbers of $\mathrm{Li}$ within these adducts. Concerning coordination numbers, as indicated in Scheme $\mathrm{I}$, dissociation of $\mathrm{Li}_{2} \mathrm{Br}_{2}$ ring dimers of 1 , in which $\mathrm{Li}$ is unusually five coordinate (as shown previously in the solid state $^{7}$ ), will afford monomers in which $\mathrm{Li}$ is still four coordinate - a coordination number towards which Li often strives (Scheme I). ${ }^{1,2}$ In contrast, dissociation of $\mathrm{Li}_{2} \mathrm{~N}_{2}$ dimers of 2 , in which $\mathrm{Li}$ is four coordinate, results in monomers (Scheme II, route A) where $\mathrm{Li}$ is only two coordinate $-\mathrm{a}$ coordinative situation in which $\mathrm{Li}$ is generally less satisfied except in very sterically crowded situations. ${ }^{1,2}$ Hence, it is understandable that dissociation of 1 occurs more readily than dissociation of 2.

Finally, some comment should be made on the low concentration measurements $\left(<0.02 \mathrm{~mol} \mathrm{~L}^{-1}\right)$ on the amidolithium complex 2 . These deviate from the simple dimer $\rightleftharpoons$ monomer behavior found for this complex at higher concentrations (Figure 6). The degree of association $(n)$ falls significantly, and reproducably, below the expected curve. This implies that another equilibrium, not involving $\mathrm{N}_{2} \mathrm{Li}_{2}$ dimer fragmentation, starts to dominate at these low concentrations. Such behavior is consistent with the loss of donor molecules (HMPA) from intact dimers of 2 , producing unsolvated dimers $\left[\left(\mathrm{C}_{6} \mathrm{H}_{11}\right)_{2} \mathrm{NLi}\right]_{2}$

(14) Best fit theoretical curves were determined by calculating the average of the equilibrium constants for the most accurate points $(\geq 0.02$ mol L-1). Using this value for $K_{\mathrm{s}}$, the theoretical relationship was then used to plot the curve (for low concentration measurements, where $\Delta T$ is small, $n$ is extremely sensitive to small changes in the measured temperature). 
together with free HMPA ligands (Scheme II, route B). Since three solution species would now be present in solution compared to only two for the complexed dimer $\rightleftharpoons$ monomer equilibrium, the value of $n$ found would be below that predicted.

\section{Conclusions}

We describe simple equations which allow cryoscopic measurements on solutions of compounds and complexes to be analyzed to give much useful information. These findings are especially relevant to reagents in solution in that they indicate with reasonable certainty the identity of equilibrium species present and quantify their relative concentrations. The key points apparent from this study are (a) prior knowledge of the species under investigation may be limited to its formula and therefore its empirical mass. No prior knowledge of the degree of association $(n)$ either in solution or in the solid state (by way of a crystal structure) is required; (b) cryoscopic measurements give the variation of degree of association with concentration. These data, when plotted, will fit best one particular derived relationship, indicative of the equilibrium present in solution; (c) such a fit thereby allows the equilibrium present in solution to be established with reasonable certainty, species present in solution to be identified, and structures to be postulated irrespective of the solid-state structure of the complex; (d) the method allows, through quantification of equilibria $\left(K_{\mathrm{s}}\right.$ and $\Delta G^{\circ}$ values), the position of equilibria at different concentrations to be determined; (e) these fundamental thermodynamic data, obtained directly from solution measurements, are of value in comparison with (gas phase) theoretical calculations. Such calculations have formed the basis of much of our current knowledge of reactive main group organometallic complexes; $1,2,15$ (f) although applied in this study to two lithium complexes, the method is general and should be applicable to any compound or complex involved in any equilibrium process in solution, the only proviso being that the solvent involved is amenable to colligative investigation.

Acknowledgment. We thank the SERC (M.G.D.,R.S., D.S.W.), the Associated Octel Co. Ltd., Ellesmere Port, U.K. (M.G.D., R.S., D.S.W.), the Nuffield Foundation (D.S.W.), the Royal Society (D.S.W.), and the DFG (D.S.). We also thank Dr. David Hussain (Cambridge, U.K.) for helpful discussions and Mr. D.C. Edwards and Mr. S.M. Fairbrother for glassblowing.

(15) See for example, (a) Armstrong, D. R.; Perkins, P. G. Coord.Chem. Rev. 1981, 38, 139. (b) Schleyer, P. v. R. Pure Appl. Chem. 1983, 55, 355; Ibid. 1984, 56, 151. (c) Streitwieser, A. Acc. Chem. Res. 1984, 17, 353. (d) Kaufmann, E.; Gose, J.; Schleyer, P. v. R. Organometallics 1989, 8, 2577. 